\title{
Keine Arroganz, aber auch keine falsche Bescheidenheit!
}

\section{Was die EU von anderen Regionalorganisationen lernen kann}

Spätestens 2010 hat die aktuelle Wirtschafts- und Finanzkrise die Europäische Union (EU) im Kern getroffen. Die Staatsschuldenkrise hat Konstruktionsfehler der europäischen Wirtschafts- und Währungsunion aufgedeckt. Darüber hinaus hat sich diese Krise wie ein Geschwür auf verschiedenste Bereiche des Gemeinschaftsgebildes ausgeweitet: Die Zustimmung zur Integration geht zurück, alte, schon vergessen geglaubte Nationalismen erleben einen neuen Frühling und in zahlreichen Ländern fordern Politiker zusammen mit großen Bevölkerungsteilen eine Renationalisierung bereits integrierter Politikfelder. Für viele Beobachter steht die EU an einem Wendepunkt und es stellt sich in der Tat die Frage, ob sich die Integrationsdynamik von einem ,immer mehr“ zu einem ,immer weniger“ umkehrt. ${ }^{1}$

Was nun, EU? Kann sich das europäische Gemeinschaftsgebilde aus sich selbst heraus reformieren? Oder kann es auf Hilfe und Anregungen von außen hoffen? Im vorliegenden Essay konzentriere ich mich auf die Beantwortung der Frage, was die EU von anderen Regionalorganisationen lernen kann.

Regionale Integration ist nicht auf die EU und nicht auf Europa beschränkt. ${ }^{2}$ Doch in aller Regel nimmt die EU nach wie vor sowohl in der vergleichenden Forschung als auch im politischen Austausch zwischen der EU und anderen Regionalorganisationen die Rolle eines „Benchmarks“ oder - kritischer betrachtet - eines Lehrmeisters ein. ${ }^{3}$ Dieser Praxis zum Trotz frage ich, ob andere Regionalorganisationen

1 Die Frage der Disintegration wird intensiver in einem jüngeren Sammelband von Eppler/Scheller (2013) diskutiert.

2 Für das Jahr 2011 zählt das Yearbook of International Organizations (2012: 25) 191 regional orientierte intergouvernementale Organisationen. Das Worldwide Governement Directory with Intergovernmental Organizations (2011) listet ungefähr 115 internationale Organisationen mit einem regionalen Fokus auf (eigene Zählung); allerdings muss angemerkt werden, dass die wenigsten dieser Organisationen ein umfassendes Aufgabenportfolio aufweisen. Börzel (2011) bietet eine nützliche Übersicht vertikaler und sektoraler Integration verschiedener Regionalorganisationen. Vergleiche einführend in zentrale Regionalorganisationen auch Börzel u. a. (2012) und Kühnhardt (2010).

3 Insbesondere Vertreter des „New Regionalism“ Ansatzes sehen dies sehr kritisch (Söderbaum/Shaw 2003; Warleigh-Lack/Robinson/Rosamond 2011; Warleigh-Lack/Rosamond 2010). 
nicht auch Vorbild für die Weiterentwicklung oder Konsolidierung der EU sein könnten.

Auch unter der Gefahr, mich dem Vorwurf der „integration snobbery“ (Murray 2010) auszusetzen, komme ich im Folgenden jedoch zu dem Befund, dass die EU aktuell nur vergleichsweise wenig vom institutionellen Setup, der Arbeitsweise, den Politikinhalten und dem Auftreten anderer Regionalorganisationen lernen kann. Dies soll keineswegs suggerieren, dass andere Regionalorganisationen alles falsch oder schlechter als die EU machen. Tatsächlich gehen sie teilweise sogar in ihren Bestrebungen über die Zielvorgaben der EU hinaus. Allerdings ist es so, dass die EU vor anderen Herausforderungen steht als andere Regionalorganisationen. Auch sind die Ausgangsbedingungen und die Zusammensetzung anderer Regionalorganisationen so unterschiedlich, dass eine Übertragung ihrer Instrumente auf die EU „eins zu eins“ “im Sinne eines „Copy \& Paste“ als kaum möglich und in vielen Fällen auch nicht unbedingt wünschbar erscheint. Das heißt aber nicht, dass der Vergleich als solcher nicht fruchtbar ist: Die in den 1990er Jahren ,wiedergeborene“ vergleichende Regionale Integrationsforschung ist eine durchaus erfreuliche Entwicklung, die unbestritten anregende empirisch-analytische Resultate liefert, nicht zuletzt, weil sie eine Möglichkeit darstellt, die EU, aber auch andere Regionalorganisationen, von dem „N=1 Fluch“ zu befreien (Börzel u. a. 2012; Choi/Caporaso 2002; Mansfield/Milner 1997; Mattli 1999). Auch aus der hier eingenommenen, auf Handlungsempfehlungen zielenden Sichtweise kann die EU aus anderen Fällen Lehren ziehen - und sei es die Erkenntnis, dass die EU ihren „Job“ vergleichsweise ordentlich macht und dass sie gut daran tut, auch ihre Werte nach Innen und Außen mit Nachdruck zu vertreten. Im Folgenden werde ich dieses Argument unter Hinzuziehung von Beispielen aus unterschiedlichen Regionalorganisationen in aller Knappheit darlegen. Ich behandele zunächst Institutionen, betrachte dann ausgewählte Policies und schließlich das Auftreten einer Regionalorganisation nach Innen und Außen.

In Bezug auf Institutionen ist das Lernpotenzial der EU sehr begrenzt, haben sich doch die meisten Regionalorganisationen bezüglich ihres institutionellen Setups an dem Vorbild der Europäischen Gemeinschaft beziehungsweise der EU orientiert (Kühnhardt 2010). So erinnern beim MERCOSUR die ,presidential summits" an den Europäischen Rat, der "Consejo del Mercado Común" entspricht einem auf Binnenmarktfragen konzentrierten Ministerrat, das Sekretariat stellt eine abgeschwächte Variante der Europäischen Kommission dar und für die im Jahr $2005 \mathrm{zu}$ einem MERCOSUR-Parlament umgewandelte „Comisión Parlamentaria Conjunta" wird das Europäische Parlament häufig als Vorbild betrachtet. Darüber hinaus besteht seit 2002 ein MERCOSUR-Gerichtshof. Ähnlich verhält es sich mit der 
Afrikanischen Union (AU). Die Schwierigkeit, von anderen Regionalorganisationen in Bezug auf den institutionellen Aufbau zu lernen, erklärt sich jedoch auch daraus, dass die Tiefe und Breite an regionaler Integration (Rittberger/Schimmelfennig (2005) würden von ,vertikaler“ und „sektoraler“ Integration sprechen), die die EU aufweist, neuartige Herausforderungen mit sich bringt, beispielsweise in Bezug auf die demokratische Qualität ihrer „Verfassung“. Bei aller teils berechtigten Kritik an Funktionsweise und Stellung des Europäischen Parlaments finden wir doch keine andere internationale Völker-Vertretung, die eine ähnliche integrative Repräsentanz in der politischen Willensbildung aufweist.

Bei den Policies wird die Angelegenheit naturgemäß komplizierter. Eine vergleichsweise gut funktionierende Währungsunion stellt die Westafrikanische Wirtschafts- und Währungsunion UEMOA (Union économique et Monétaire Ouest Africaine) dar, die sich auch um eine Konvergenz makroökonomischer Politiken der Mitgliedstaaten bemüht. Ich möchte hier jedoch ein Politikfeld herausgreifen, bei dem die EU einigen Regionalorganisationen hinterherhinkt, nämlich der Sicherheits- und Verteidigungspolitik. Hier verhielt sich Westeuropa - wohlgemerkt unter dem Schutzschirm der NATO - lange Zeit recht zurückhaltend, ganz im Sinne von Stanley Hoffmanns (1966) intergouvernementalistischer Erwartung, dass weitreichende Integration in stärker souveränitätsbezogenen Politikbereichen unwahrscheinlich ist. Bei „high politics“ hört für Hoffmann (1966: 882) der Spaß auf: „Russian roulette is fine only as long as the gun is filled with blanks". Maastricht und die Entwicklungen in der Folge des britisch-französischen Gipfels Saint Malo stellen sicherlich gewisse Integrationsfortschritte im Bereich der Außen- und Sicherheitspolitik dar, doch fällt auf, dass Anspruch und Wirklichkeit in diesem Politikfeld nach wie vor oft auseinanderklaffen. Man denke an die Schwierigkeiten der Umsetzung der in Helsinki 1999 geplanten „EU Rapid Reaction Force“. Und auch die jüngeren Ereignisse in Mali und der Zentralafrikanischen Republik illustrieren, dass in Fragen von Militäreinsätzen eine Einigung zwischen den Mitgliedstaaten der EU nur schwer zu erzielen ist. Wenn es zu einer Einigung kommt, erfolgt diese gewöhnlich auf einem vergleichsweise niedrigen Niveau. Demgegenüber ist bemerkenswert, dass sowohl die Afrikanische Union (AU) als auch die Westafrikanische Wirtschaftsgemeinschaft ECOWAS über strukturierte militärische Handlungsinstrumente verfügen und von diesen auch Gebrauch machen (Neethling 2005; Striebinger 2012). Die „ECOWAS Standby Force“ kann im Rahmen des „ECOWAS conflict prevention framework" friedenserhaltend und friedensschaffend aktiv werden. Und im Rahmen der „African Peace and Security Architecture“verfügt die AU über eine regional organisierte „African Standby Force“. In Artikel 4 der Konstituierenden Akte der AU wird nicht nur die Schaffung einer gemeinsamen Verteidi- 
gungspolitik für den gesamten afrikanischen Kontinent angestrebt, sondern dort ist auch das Recht der Gemeinschaft festgelegt, "to intervene in a Member State pursuant to a decision of the Assembly in respect of grave circumstances, namely: war crimes, genocide and crimes against humanity [...]", sowie "the right of Member States to request intervention from the Union in order to restore peace and security [...]." Sowohl die ECOWAS als auch die AU verfügen also über weiterreichende Durchgriffsmöglichkeiten auf die Mitgliedstaaten als die EU. Darüber hinaus haben sie sich in der Vergangenheit als entscheidungsfreudiger beim Einsatz von Streitkräften erwiesen. Jenseits der politischen Frage nach der Wünschbarkeit eines stärkeren sicherheitspolitischen Engagements der EU lässt sich also feststellen, dass bei den genannten afrikanischen Regionalorganisationen Anspruch und Wirklichkeit zumindest in der Frage der Sicherheits- und Verteidigungspolitik etwas stärker übereinzustimmen scheinen. Das heißt freilich nicht, dass auch die afrikanischen Partner immer ihre Ziele erreichen: Im Falle des Konfliktes in Mali wäre die ECOWAS ohne das Eingreifen der ehemaligen Kolonialmacht Frankreich weitestgehend handlungsunfähig geblieben. Aber auch der Verband südostasiatischer Nationen ASEAN hat zum Teil weniger große Schwierigkeiten als die EU, geschlossen nach Außen aufzutreten. Beispielsweise in Bezug auf den Abschluss von Freihandelsabkommen scheint die ASEAN handlungsfähiger, besitzt dieser Verband doch Freihandelsabkommen mit China, Indien, Südkorea, Australien und Neuseeland.

In der EU wird durch Artikel 7 des Lissabonner Vertrages festgelegt, dass der „Rat mit qualifizierter Mehrheit beschließen [kann], bestimmte Rechte auszusetzen, die sich aus der Anwendung der Verträge auf den betroffenen Mitgliedstaat herleiten, einschließlich der Stimmrechte des Vertreters der Regierung dieses Mitgliedstaats im Rat" für den Fall, dass ein Mitgliedstaat, die in Artikel 2 des EU-Vertrages genannten Werte schwerwiegend und anhaltend verletzt. Beim Schutz der Demokratie kann die EU jedoch insofern keine Pionierrolle oder ein Alleinstellungsmerkmal beanspruchen, hatte sich die Organisation Amerikanischer Staaten (OAS) doch schon im Jahre 1948 der Demokratie verpflichtet (Heine/Weiffen 2014). Und auch andere Regionalverträge enthalten Demokratie- oder Stabilitätsklauseln (van Hüllen/Börzel 2013). So hat der MERCOSUR durch die Protokolle von Ushuaia I und II Sanktionsmechanismen formuliert, die bis zu einem Ausschluss eines Landes aus dem Regionalbündnis reichen. ${ }^{4}$ Anwendung fand diese Klausel, obgleich noch nicht vollständig ratifiziert, im Jahre 2012: Nach der Absetzung von Staatspräsident Fernando Lugo setzte der MERCOSUR die Mitgliedschaft Paraguays mit dem Ver-

4 Vergleiche etwa Artikel 6 des Protocolo de Montevideo sobre compromiso con la democracia en el Mercosur, genannt Ushuaia II. 
weis auf die Gefahren für die paraguayische Demokratie aus (Beschlüsse des Gipfels von Mendoza vom 31.7.2012). Etwas nachdenklich sollte in diesem Zusammenhang jedoch stimmen, dass der MERCOSUR zwar die Mitgliedschaft Paraguays aussetzte, gleichzeitig aber den Beitritt Venezuelas vollzog - obgleich das paraguayische Parlament zuvor die Vollmitgliedschaft der bolivarischen Republik gerade wegen ihrer mangelhaften Demokratie ablehnt hatte. Aus dem Falle Paraguays generell zu schließen, die EU könne vom MERCOSUR die Verteidigung von Demokratie erlernen, erscheint daher als etwas überzogen.

Positiv gewendet unterstreichen diese Beispiele jedoch auch, dass die EU selbstbewusst zu ihrer eigenen, wertegebundenen Politik stehen kann. Im Umgang mit jüngeren demokratischen Krisen wie in Ungarn oder Rumänien oder auch mangelhafter Korruptionsbekämpfung und/oder Regelbefolgung in Ländern wie Bulgarien und Kroatien empfiehlt sich eben kein Werterelativismus. Und eine Einmischung in innenpolitische Belange von Mitgliedstaaten ist keine Marotte des „sanften Monsters Brüssel“ (Enzensberger 2011), sondern gängige Praxis in verschiedenen funktionierenden Regionalorganisationen.

Dies bringt mich zu einem letzten Punkt. Befragt man Regionalexperten zu den Lektionen, die die EU von anderen Regionalorganisationen lernen könnte, wird geradezu reflexhaft der Vorwurf der Arroganz formuliert: Die EU solle den anderen Regionalorganisationen endlich ,,auf Augenhöhe“ begegnen und sich nicht weiter als „Nabel der Welt“ betrachten. Wenn die EU als arrogante Macht - übrigens ganz im Gegensatz zum Selbstbild der ,normative power“ (Manners 2002) - wahrgenommen wird, kann das ihre Handlungsoptionen einschränken und insbesondere die Kooperation bei der Lösung politischer, ökonomischer und humanitärer Herausforderungen erschweren. Das Argument würde dann lauten, dass eine weniger „,arrogante“ EU auf weniger Ablehnung stoßen und ihre Ziele in Zusammenarbeit mit anderen Regionalorganisationen in einer interdependenten Welt besser erreichen könne. Und doch stellt sich hier gleichsam die Frage, was genau mit „Arroganz" gemeint ist. Soll die EU ihre Werte weniger verallgemeinern und sich weniger in Belange anderer Regionen einmischen? Was bedeutet das für die menschenrechtlichen Maßstäbe? Fällt eine Ablehnung der Zusammenarbeit mit Autokraten wie Robert Mugabe unter „Arroganz“? Nach meinem Dafürhalten darf der Vorwurf der Arroganz nicht einem Werterelativismus Tor und Tür öffnen. Im Gegenteil: Die EU darf und soll ihre Werte in der Welt vertreten!

Die EU ist nach wie vor das wichtigste Regionalbündnis der Welt. Keine andere Regionalorganisation kommt der EU in Bezug auf wirtschaftlichen Wohlstand, Entwicklung und Demokratie auch nur nah (Moravcsik 2009). Das heißt freilich nicht, dass sich auch die EU nicht ständig erneuern muss und dass der Blick in andere 
Staaten oder Regionalorganisationen schadet. Die skizzenhafte Analyse anderer Regionalorganisationen legt jedoch dar, dass andere Regionalorganisationen eventuell mit Ausnahme in Bezug auf die Sicherheits- und Verteidigungspolitik kaum Modelle aufzeigen, wie aktuelle Herausforderungen der EU zu meistern sind. So kann Solidarität und die Bereitschaft zur Umverteilung, die Staaten und ihre Bürger in Zeiten der Krise zusammenhält, nicht von anderen Regionalorganisationen gelernt werden. Hier empfiehlt sich eher der Blick in Praktiken und Institutionen von Föderalstaaten. Diese Diagnose ist insofern beruhigend, als dass die EU ihre Aufgabe insgesamt so schlecht nicht zu machen scheint. Gleichzeitig unterstreicht sie einmal mehr auch die grundsätzliche Schwierigkeit erfolgreicher regionaler Integration. In einer solchen Sichtweise bleibt die EU eher ein historischer Sonderfall, den es nicht nur aus Gründen des „Artenschutzes“ umso dringlicher zu verteidigen gilt. $^{5}$

\section{Literatur}

Börzel, Tanja A., 2011: Comparative Regionalism: A New Research Agenda, in: Kolleg-Forschergruppe Working Paper Series ,The Transformative Power of Europe“, Freie Universität Berlin.

Börzel, Tanja A./Goltermann, Lukas/Lohaus, Mathis/Striebinger, Kai (Hrsg.), 2012: Roads to Regionalism. Genesis, Design and Effects of Regional Organizations, Aldershot.

Choi, Young Jong/Caporaso, James A., 2002: Comparative Regional Integration, in: Walter Carlsnaes/Thomas Risse/Beth A. Simmons (Hrsg.), Handbook of International Relations, London, 480-499.

Enzensberger, Hans Magnus, 2011: Sanftes Monster Brüssel oder die Entmündigung Europas, Frankfurt a. M.

Eppler, Annegret/Scheller, Henrik (Hrsg.), 2013: Zur Konzeptionalisierung europäischer Desintegration. Zug- und Gegenkräfte im europäischen Integrationsprozess, Baden-Baden.

Heine, Jorge/Weiffen, Brigitte, 2014: Twenty-first Century Democracy Promotion in the Americas, London (i. E.).

Hoffmann, Stanley, 1966: Obstinate or Obsolete? The Fate of the Nation State and the Future of Western Europe, in: Daedalus 95 (4), 861-898.

Kühnhardt, Ludger, 2010: Region-Building. The Global Proliferation of Regional Integration (Vol. I), New York.

5 Für Anregungen und hilfreiche Diskussionen danke ich Hanno Degner, Esther Leuffen, Kerstin Radtke, Sophia Schemel, Brigitte Weiffen und Martin Welz. 
Keine Arroganz, aber auch keine falsche Bescheidenheit!

Manners, Ian, 2002: Normative Power Europe: A Contradiction in Terms?, in: Journal of Common Market Studies 40 (2), 235-258.

Mansfield, Edward D./Milner, Helen V. (Hrsg.), 1997: The Political Economy of Regionalism, New York.

Mattli, Walter, 1999: The Logic of Regional Integration. Europe and Beyond. Cambridge u. a.

Moravcsik, Andrew, 2009: Europe: The Quiet Superpower, in: French Politics 7 (3/4), 403-422.

Murray, Philomena, 2010: Comparative regional integration in the EU and East Asia: Moving beyond integration snobbery, in: International Politics 47, 308-323.

Neethling, Theo, 2005: Realizing the African Standby Force as a Pan-African Ideal: Progress, Prospects and Challenges, in: Journal of Military and Strategic Studies 8 (1), 1-25.

Rittberger, Berthold/Schimmelfennig, Frank, 2005: Integrationstheorien: Entstehung und Entwicklung der EU, in: Katharina Holzinger/Christoph Knill/Dirk Peters/Berthold Rittberger/Frank Schimmelfennig/Wolfgang Wagner (Hrsg.), Die Europäische Union. Theorien und Analysekonzepte, Paderborn.

Söderbaum, Fredrik/Shaw, Timothy M., 2003: Theories of New Regionalism: A Palgrave Reader, Houndmills.

Striebinger, Kai, 2012: When Pigs Fly: ECOWAS and the Protection of Constitutional Order in Events of Coups d'Etat, in: Tanja A. Börzel/Lukas Goltermann/ Mathis Lohaus/Kai Striebinger (Hrsg.), Roads to Regionalism. Genesis, Design, and Effects of Regional Organizations, Aldershot, 179-196.

van Hüllen, Vera/Börzel, Tanja A., 2013: The EU's Governance Transfer. From External Promotion to Internal Protection?, SFB-Governance Working Paper Series, Working Paper 56.

Warleigh-Lack, Alex/Robinson, Nick/Rosamond, Ben (Hrsg.), 2011: New Regionalism and the European Union: Dialogues, Comparisons and New Research Directions, London.

Warleigh-Lack, Alex/Rosamond, Ben, 2010: Across the EU Studies - New Regionalism Frontier: Invitation to a Dialogue, in: Journal of Common Market Studies 48 (4), 993-1013. 
Dirk Leuffen

Korrespondenzanschrift:

Prof. Dr. Dirk Leuffen

Universität Konstanz

Fachbereich Politik- und Verwaltungswissenschaft

Lehrstuhl für Politikwissenschaft mit dem Schwerpunkt Internationale Politik

Postfach 83

78457 Konstanz

E-Mail: Dirk.Leuffen@uni-konstanz.de 